PROCEEDINGS OF THE

AMERICAN MATHEMATICAL SOCIETY

Volume 135, Number 9, September 2007, Pages 2811-2819

S 0002-9939(07)08796-5

Article electronically published on May 2, 2007

\title{
MOMENT PROBLEM WITH CONTRACTIVE SOLUTIONS: THE REGULAR CASE
}

\author{
ZENON J. JABŁOŃSKI
}

(Communicated by Joseph A. Ball)

\begin{abstract}
An operator multivariate moment problem with contractive solutions having regular unitary dilation is characterized in terms of the initial data. This extends a recent result of Sebestyén and Popovici, but the ideas of our proof differ from those used by them. The connection between the operator multivariate moment problem and harmonizable multivariate discrete processes is mentioned.
\end{abstract}

1. From now on $\mathcal{D}$ stands for a complex inner product space and $\mathcal{H}$ for a complex Hilbert space. Let us denote by $\boldsymbol{L}(\mathcal{D}, \mathcal{H})($ resp. $\boldsymbol{B}(\mathcal{D}, \mathcal{H})$ ) the set of all linear (resp. bounded linear) operators from $\mathcal{D}$ to $\mathcal{H}$. For simplicity we write $\boldsymbol{B}(\mathcal{H})$ instead of $\boldsymbol{B}(\mathcal{H}, \mathcal{H}) ; I_{\mathcal{D}}$ stands for the identity operator on $\mathcal{D}$. For $T \in \boldsymbol{L}(\mathcal{D}, \mathcal{H})$, we set $\mathcal{R}(T)=T(\mathcal{D})$.

Let $\mathbb{Z}$ stand for the additive group of all integers. Assume that $\Omega$ is a nonempty set. Denote by $\mathbb{Z}[\Omega]$ the group which is the direct sum of $\operatorname{card}(\Omega)$ copies of the additive group $\mathbb{Z}$; that is, $\mathbb{Z}[\Omega]$ is the set of all functions $\boldsymbol{m}: \Omega \rightarrow \mathbb{Z}$ of finite support $\{\alpha \in \Omega: \boldsymbol{m}(\alpha) \neq 0\}$ equipped with a pointwise defined group operation. The zero function is a neutral element of $\mathbb{Z}[\Omega]$. If $\Xi$ is a subset of $\Omega$, then we can think of $\mathbb{Z}[\Xi]$ as a subset of $\mathbb{Z}[\Omega]$.

Let

$$
\mathbb{Z}[\Omega]_{+}=\{\boldsymbol{m} \in \mathbb{Z}[\Omega] ; \boldsymbol{m}(\alpha) \geqslant 0 \text { for all } \alpha \in \Omega\} .
$$

For $n \in \mathbb{Z}$, we set $n^{+}=\max \{n, 0\}$ and $n^{-}=-\min \{n, 0\}$. Given $\boldsymbol{m} \in \mathbb{Z}[\Omega]$, we denote by $\boldsymbol{m}^{+}, \boldsymbol{m}^{-} \in \mathbb{Z}[\Omega]_{+}$the positive and the negative parts of $\boldsymbol{m}$, i.e.,

$$
\begin{array}{ll}
\boldsymbol{m}^{+}(\alpha) \stackrel{\mathrm{df}}{=}(\boldsymbol{m}(\alpha))^{+}, \quad \alpha \in \Omega, \\
\boldsymbol{m}^{-}(\alpha) \stackrel{\mathrm{df}}{=}(\boldsymbol{m}(\alpha))^{-}, \quad \alpha \in \Omega .
\end{array}
$$

For a finite subset $v$ of $\Omega$, we define the function $\boldsymbol{e}_{v} \in \mathbb{Z}[\Omega]_{+}$by

$$
\boldsymbol{e}_{v}(\beta)= \begin{cases}1 & \text { if } \beta \in v \\ 0 & \text { if } \beta \notin v\end{cases}
$$

Received by the editors December 29, 2005 and, in revised form, May 18, 2006.

2000 Mathematics Subject Classification. Primary 47A20, 60G60; Secondary 44A60, 47A57, $60 \mathrm{G} 10$.

Key words and phrases. Systems of contractions with regular unitary dilations, vector and operator moment problems, harmonizable multivariate discrete processes.

This work was supported by the KBN grant 2 P03A 037024.

(C)2007 American Mathematical Society 
and set $\boldsymbol{e}_{\alpha}=\boldsymbol{e}_{\{\alpha\}}$ for $\alpha \in \Omega$.

Let $\boldsymbol{T}=\left\{T_{\alpha}\right\}_{\alpha \in \Omega} \subset \boldsymbol{B}(\mathcal{H})$ be a family of commuting operators. Set

$$
\boldsymbol{T}^{\boldsymbol{m}}=\prod_{\alpha \in \Omega} T_{\alpha}^{\boldsymbol{m}(\alpha)}, \quad \boldsymbol{m} \in \mathbb{Z}[\Omega]_{+} .
$$

We say that $\boldsymbol{T}$ has a unitary dilation if there exists a complex Hilbert space $\mathcal{K} \supset \mathcal{H}$ (isometric embedding) and a family $\boldsymbol{U}=\left\{U_{\alpha}\right\}_{\alpha \in \Omega} \subset \boldsymbol{B}(\mathcal{K})$ of commuting unitary operators such that

$$
\boldsymbol{T}^{\boldsymbol{m}}=\left.P \boldsymbol{U}^{\boldsymbol{m}}\right|_{\mathcal{H}}, \quad \boldsymbol{m} \in \mathbb{Z}[\Omega]_{+},
$$

where $P$ is the orthogonal projection of $\mathcal{K}$ onto $\mathcal{H}$. If moreover

$$
\left(\boldsymbol{T}^{\boldsymbol{m}^{-}}\right)^{*} \boldsymbol{T}^{\boldsymbol{m}^{+}}=\left.P \boldsymbol{U}^{\boldsymbol{m}}\right|_{\mathcal{H}}, \quad \boldsymbol{m} \in \mathbb{Z}[\Omega],
$$

then we say that $\boldsymbol{T}$ has a regular unitary dilation. Regular dilations were introduced by Brehmer [1]. Let us recall that this type of dilations has been studied in [19, 7 , 2, 3, 5, 6, 21, 4, 10, 11, 12 .

Let $X$ be an arbitrary nonempty set. An operator moment problem considered in this paper entails determining whether, for a given family $\left\{A_{m}^{x}\right\}_{\substack{x \in \mathbb{Z}[\Omega]_{+}}} \subset \boldsymbol{L}(\mathcal{D}, \mathcal{H})$ of operators, there exists a family $\boldsymbol{T}=\left\{T_{\alpha}\right\}_{\alpha \in \Omega} \subset \boldsymbol{B}(\mathcal{H})$ of commuting contractions having a regular unitary dilation and such that

$$
A_{\boldsymbol{m}}^{x}=\boldsymbol{T}^{\boldsymbol{m}} A_{\mathbf{0}}^{x}, \quad \boldsymbol{m} \in \mathbb{Z}[\Omega]_{+}, x \in X .
$$

The moment problem (11) has been considered and solved by Popovici [12] (see also [11] for the vector case) in the case that the operator data $\left\{A_{m, n}^{x}\right\} \underset{(m, n) \in \mathbb{Z}^{2}}{\substack{x \in X \\ \text { and }}}$ are a bounded double-indexed sequence (see also [15, 16, 4, 17, in the case in which $\operatorname{card}(X)=1$ ). The multidimensional vector moment problem with $\operatorname{card}(X)=1$ has been solved by Z. Sebestyén and Popovici in [13. By contrast to the method used in [12, which relies on the results of [15, 4] and does not seem to be easily generalizable to the case of an arbitrary family of operators, our technique (cf. proof of Theorem 4) enables us to fully solve problem (11).

It turns out that the moment problem (1) is connected with the concept of so-called weak operator harmonizability 11 of regular type. More precisely, if $\boldsymbol{T}=$ $\left\{T_{\alpha}\right\}_{\alpha \in \Omega} \subset \boldsymbol{B}(\mathcal{H})$ is a family of commuting contractions having a regular unitary dilation and $A_{\mathbf{0}} \in \boldsymbol{L}(\mathcal{D}, \mathcal{H})$, then the process $\left\{\boldsymbol{T}^{\boldsymbol{m}} A_{\mathbf{0}}\right\}_{\boldsymbol{m} \in \mathbb{Z}[\Omega]_{+}}$is weakly operator harmonizable of regular type (cf. [12]; see also [9, 8] for more details). Hence, if our problem attached to the family $\left\{A_{\boldsymbol{m}}^{x}\right\} \underset{\boldsymbol{m} \in \mathbb{Z}[\Omega]_{+}}{x \in X} \subset \boldsymbol{L}(\mathcal{D}, \mathcal{H})$ has a solution, then each process in the family is weakly operator harmonizable of regular type.

2. In what follows, we write $\mathcal{D}[\Xi]$ for the set of all maps $f: \Xi \rightarrow \mathcal{D}$ with finite support ( $\Xi$ is an arbitrary nonempty set).

The following lemma characterizes families of operators having regular unitary dilations.

Lemma 1 ([1]; see also [7, 20]). Let $\boldsymbol{T}=\left\{T_{\alpha}\right\}_{\alpha \in \Omega} \subseteq \boldsymbol{B}(\mathcal{H})$ be a commuting family of operators. Then the following conditions are equivalent:

(a) $\boldsymbol{T}$ has a regular unitary dilation;

\footnotetext{
${ }^{1}$ The notion of a harmonizable process has been introduced in [14] by Rozanov.
} 
(b) for any map $f \in \mathcal{H}\left[\mathbb{Z}[\Omega]_{+}\right]$the following inequality holds 2

$$
\sum_{\boldsymbol{m}, \boldsymbol{n} \in \mathbb{Z}[\Omega]_{+}}\left\langle\boldsymbol{T}^{(\boldsymbol{m}-\boldsymbol{n})^{+}} f(\boldsymbol{m}), \boldsymbol{T}^{(\boldsymbol{m}-\boldsymbol{n})^{-}} f(\boldsymbol{n})\right\rangle \geqslant 0 ;
$$

(c) for any finite subset $u$ of $\Omega$ and for any vector $f \in \mathcal{H}$ the following inequality holds:

$$
\sum_{v \subseteq u}(-1)^{|v|}\left\|\boldsymbol{T}^{\boldsymbol{e}_{v}} f\right\|^{2} \geqslant 0
$$

where $|v|$ denotes the number of elements of $v$.

Given a family $\left\{A_{\boldsymbol{m}}^{x}\right\} \underset{\boldsymbol{m} \in \mathbb{Z}[\Omega]_{+}}{x \in X} \subset \boldsymbol{L}(\mathcal{D}, \mathcal{H})$, we set

$$
\mathcal{R}_{\mathrm{A}}=\operatorname{lin} \bigcup_{\substack{x \in X \\ m \in \mathbb{Z}[\Omega]_{+}}} A_{\boldsymbol{m}}^{x}(\mathcal{D}), \quad \mathcal{H}_{\mathrm{A}}=\overline{\mathcal{R}}_{\mathrm{A}} .
$$

The next lemma is an extension of [17, Theorem 3.1] and [8, Lemma 4] to the arbitrary nonempty index set $X$. It may be proved in a similar way as 8 , Lemma 4]. However, for the convenience of the reader, we give the proof of this lemma for our case.

Lemma 2. Let $\left\{A_{\boldsymbol{m}}^{x}\right\}_{\substack{\boldsymbol{m} \in \mathbb{Z}[\Omega]_{+} \\ x \in X}} \subseteq \boldsymbol{L}(\mathcal{D}, \mathcal{H})$. Then for every $\alpha \in \Omega$ the following conditions are equivalent:

(i) there exists a contraction $T_{\alpha} \in \boldsymbol{B}(\mathcal{H})$ such that

$$
T_{\alpha} A_{\boldsymbol{m}}^{x}=A_{\boldsymbol{e}_{\alpha}+\boldsymbol{m}}^{x}, \quad \boldsymbol{m} \in \mathbb{Z}[\Omega]_{+}, x \in X ;
$$

(ii) for any integer $c \geqslant 1$ and for all maps $h_{1}, \ldots, h_{c} \in \mathcal{D}\left[\mathbb{Z}[\Omega]_{+} \times X\right]$,

$$
\sum_{k, l=1}^{c} \sum_{\substack{x, y \in X \\ \boldsymbol{m}, \boldsymbol{n} \in \mathbb{Z}[\Omega]_{+}}}\left\langle A_{(k-l)+\boldsymbol{e}_{\alpha}+\boldsymbol{m}}^{x} h_{k}(\boldsymbol{m}, x), A_{(k-l)-\boldsymbol{e}_{\alpha}+\boldsymbol{n}}^{y} h_{l}(\boldsymbol{n}, y)\right\rangle \geqslant 0
$$

(ii') for all maps $h_{1}, h_{2} \in \mathcal{D}\left[\mathbb{Z}[\Omega]_{+} \times X\right]$,

$$
\sum_{k, l=1}^{2} \sum_{\substack{x, y \in X \\ \boldsymbol{m}, \boldsymbol{n} \in \mathbb{Z}[\Omega]_{+}}}\left\langle A_{(k-l)+\boldsymbol{e}_{\alpha}+\boldsymbol{m}}^{x} h_{k}(\boldsymbol{m}, x), A_{(k-l)-\boldsymbol{e}_{\alpha}+\boldsymbol{n}}^{y} h_{l}(\boldsymbol{n}, y)\right\rangle \geqslant 0
$$

(iii) for any map $h \in \mathcal{D}\left[\mathbb{Z}[\Omega]_{+} \times X\right]$,

$$
\left\|\sum_{\substack{x \in X \\ \boldsymbol{m} \in \mathbb{Z}[\Omega]_{+}}} A_{\boldsymbol{m}+\boldsymbol{e}_{\alpha}}^{x} h(\boldsymbol{m}, x)\right\| \leqslant\left\|\sum_{\substack{x \in X \\ \boldsymbol{m} \in \mathbb{Z}[\Omega]_{+}}} A_{\boldsymbol{m}}^{x} h(\boldsymbol{m}, x)\right\| .
$$

Moreover, if any of the conditions (ii), (ii'), (iii) holds for every $\alpha \in \Omega$, then there exists a family $\left\{T_{\alpha}\right\}_{\alpha \in \Omega} \subset \boldsymbol{B}(\mathcal{H})$ of commuting contractions satisfying (2).

Proof. (i) $\Rightarrow\left(\right.$ ii). Let $T_{\alpha} \in \boldsymbol{B}(\mathcal{H})$ be a contraction satisfying (2). Since the map

$$
\mathbb{Z} \ni n \rightarrow \begin{cases}T_{\alpha}^{n} & \text { if } n \geq 0 \\ T_{\alpha}^{*|n|} & \text { if } n<0\end{cases}
$$

\footnotetext{
2 The condition (b) is equivalent to requiring that the form $\omega: \mathbb{Z}[\Omega] \times \mathcal{H} \times \mathcal{H} \ni(\boldsymbol{n}, f, g) \rightarrow$ $\left\langle\left(\boldsymbol{T}^{n^{-}}\right)^{*} \boldsymbol{T}^{n^{+}} f, g\right\rangle \in \mathbb{C}$ is positive definite.
} 
is positive definite on $(\mathbb{Z},+$ ) (see [20, I.8.1]), for a fixed integer $c \geqslant 1$ and maps $h_{1}, \ldots, h_{c} \in \mathcal{D}\left[\mathbb{Z}[\Omega]_{+} \times X\right]$, we get

$$
\begin{aligned}
0 & \leq \sum_{k, l=1}^{c}\left\langle T_{\alpha}^{(k-l)^{+}} \sum_{\substack{x \in X \\
\boldsymbol{m}, \in \mathbb{Z}[\Omega]_{+}}} A_{\boldsymbol{m}}^{x} h_{k}(\boldsymbol{m}, x), T_{\alpha}^{(k-l)^{-}} \sum_{\substack{y \in X \\
\boldsymbol{n} \in \mathbb{Z}[\Omega]_{+}}} A_{\boldsymbol{n}}^{y} h_{l}(\boldsymbol{n}, y)\right\rangle \\
& =\sum_{k, l=1}^{c} \sum_{\substack{x, y \in X \\
\boldsymbol{m}, \boldsymbol{n} \in \mathbb{Z}[\Omega]_{+}}}\left\langle A_{(k-l)+\boldsymbol{e}_{\alpha}+\boldsymbol{m}}^{x} h_{k}(\boldsymbol{m}, x), A_{(k-l)^{-} \boldsymbol{e}_{\alpha}+\boldsymbol{n}}^{y} h_{l}(\boldsymbol{n}, y)\right\rangle .
\end{aligned}
$$

The implication (ii) $\Rightarrow\left(\right.$ ii $\left.^{\prime}\right)$ is obvious.

$\left(\mathrm{ii}^{\prime}\right) \Rightarrow(\mathrm{iii})$. The inequality (3) can be rewritten in the following form:

$$
\left\|g_{1}\right\|^{2}+\left\|g_{2}\right\|^{2}+2 \mathfrak{R e}\left\langle g_{1}, \hat{g}_{2}\right\rangle \geqslant 0
$$

where $g_{j} \stackrel{\text { df }}{=} \sum_{\substack{x \in X \\ \boldsymbol{m} \in \mathbb{Z}[\Omega]_{+}}} A_{\boldsymbol{m}}^{x} h_{j}(\boldsymbol{m}, x)$ and $\hat{g}_{2} \stackrel{\mathrm{df}}{=} \sum_{\substack{x \in X \\ \boldsymbol{m} \in \mathbb{Z}[\Omega]_{+}}} A_{\boldsymbol{e}_{\alpha}+\boldsymbol{m}}^{x} h_{2}(\boldsymbol{m}, x)$. Set $h \in$ $\mathcal{D}\left[\mathbb{Z}[\Omega]_{+} \times X\right]$ and define $h_{1}, h_{2} \in \mathcal{D}\left[\mathbb{Z}[\Omega]_{+} \times X\right]$ by $h_{2}=h$ and

$$
h_{1}(\boldsymbol{m}, x)=\left\{\begin{array}{cl}
-h\left(\boldsymbol{m}-\boldsymbol{e}_{\alpha}, x\right) & \text { if } \boldsymbol{m}-\boldsymbol{e}_{\alpha} \in \mathbb{Z}[\Omega]_{+} \\
0 & \text { otherwise. }
\end{array}\right.
$$

A short computation shows that $g_{1}=-\hat{g}_{2}$. This and (5) yield $\left\|\hat{g}_{2}\right\|^{2} \leqslant\left\|g_{2}\right\|^{2}$, which implies (4).

(iii) $\Rightarrow(\mathrm{i})$. By virtue of (iii) there exists a unique contraction $\widetilde{T}_{\alpha} \in \boldsymbol{B}\left(\mathcal{H}_{\mathrm{A}}\right)$ such that $\widetilde{T}_{\alpha} A_{\boldsymbol{m}}^{x} f=A_{\boldsymbol{e}_{\alpha}+\boldsymbol{m}}^{x} f$ for all $\boldsymbol{m} \in \mathbb{Z}[\Omega]_{+}, x \in X$ and $f \in \mathcal{D}$. Define the operator $T_{\alpha} \in \boldsymbol{B}(\mathcal{H})$ by $T_{\alpha}=\widetilde{T}_{\alpha} \oplus I_{\mathcal{H} \ominus \mathcal{H}_{\mathrm{A}}}$. Then $T_{\alpha}$ is a contraction, which satisfies (2). Moreover, if this is done for every $\alpha \in \Omega$, then the operators $T_{\alpha}, \alpha \in \Omega$, commute. This completes the proof.

Remark 3. Regarding Lemma 2 (as well as Theorem 4 and Corollary 5), one can check that if $\mathcal{H}_{\mathrm{A}}=\mathcal{H}$, then there exists at most one operator $T_{\alpha} \in \boldsymbol{B}(\mathcal{H})$ satisfying (2). On the other hand, if there exists exactly one operator $T_{\alpha} \in \boldsymbol{B}(\mathcal{H})$ satisfying (22), then $\mathcal{H}_{\mathrm{A}}=\mathcal{H}$.

3. We are now ready to prove the main result of the paper.

Theorem 4. Suppose we are given a family $\left\{A_{\boldsymbol{m}}^{x}\right\}_{\substack{x \in X \\ \boldsymbol{m} \in \mathbb{Z}[\Omega]_{+}}} \subset \boldsymbol{L}(\mathcal{D}, \mathcal{H})$. Then the following conditions are equivalent:

(i) there exists a family $\boldsymbol{T}=\left\{T_{\alpha}\right\}_{\alpha \in \Omega} \subset \boldsymbol{B}(\mathcal{H})$ of commuting contractions having a regular unitary dilation and such that (1) holds:

(ii) for any map $h \in \mathcal{D}\left[\mathbb{Z}[\Omega]_{+} \times \mathbb{Z}[\Omega]_{+} \times X\right]$ the following inequality holds:

$$
\sum_{x, y \in X} \sum_{\substack{\boldsymbol{m}, \boldsymbol{n} \in \mathbb{Z}[\Omega]_{+} \\ \boldsymbol{s}, \boldsymbol{t} \in \mathbb{Z}[\Omega]_{+}}}\left\langle A_{(\boldsymbol{m}-\boldsymbol{n})^{+}+\boldsymbol{s}}^{x} h(\boldsymbol{m}, \boldsymbol{s}, x), A_{(\boldsymbol{m}-\boldsymbol{n})^{-+}+\boldsymbol{t}}^{y} h(\boldsymbol{n}, \boldsymbol{t}, y)\right\rangle \geqslant 0 ;
$$

(iii) for any map $h \in \mathcal{D}\left[\mathbb{Z}[\Omega]_{+} \times X\right]$ and for any finite subset $u$ of $\Omega$ the following inequality holds:

$$
\sum_{v \subseteq u}(-1)^{|v|}\left\|\sum_{\substack{x \in X \\ \boldsymbol{m} \in \mathbb{Z}[\Omega]_{+}}} A_{\boldsymbol{e}_{v}+\boldsymbol{m}}^{x} h(\boldsymbol{m}, x)\right\|^{2} \geqslant 0 .
$$


Before going to the proof, it is worth noting that the above theorem extends [12, Theorem 3.1], but the ideas of our proof differ from those used by Popovici.

Proof. (i) $\Rightarrow$ (ii). Take $h \in \mathcal{D}\left[\mathbb{Z}[\Omega]_{+} \times \mathbb{Z}[\Omega]_{+} \times X\right]$. Define $f \in \mathcal{H}\left[\mathbb{Z}[\Omega]_{+}\right]$by

$$
f(\boldsymbol{m})=\sum_{\substack{x \in X \\ s \in \mathbb{Z}[\Omega]_{+}}} A_{\boldsymbol{s}}^{x} h(\boldsymbol{m}, \boldsymbol{s}, x), \quad \boldsymbol{m} \in \mathbb{Z}[\Omega]_{+} .
$$

Applying the implication (a) $\Rightarrow$ (b) of Lemma 1 and using (11) we get

$$
\begin{aligned}
0 & \leqslant \sum_{\boldsymbol{m}, \boldsymbol{n} \in \mathbb{Z}[\Omega]_{+}}\left\langle\boldsymbol{T}^{(\boldsymbol{m}-\boldsymbol{n})^{+}} f(\boldsymbol{m}), \boldsymbol{T}^{(\boldsymbol{m}-\boldsymbol{n})^{-}} f(\boldsymbol{n})\right\rangle \\
& =\sum_{\boldsymbol{m}, \boldsymbol{n} \in \mathbb{Z}[\Omega]_{+}}\left\langle\boldsymbol{T}^{(\boldsymbol{m}-\boldsymbol{n})^{+}} \sum_{\substack{x \in X \\
s \in \mathbb{Z}[\Omega]_{+}}} A_{\boldsymbol{s}}^{x} h(\boldsymbol{m}, \boldsymbol{s}, x), \boldsymbol{T}^{(\boldsymbol{m}-\boldsymbol{n})^{-}} \sum_{\substack{y \in X \\
t \in \mathbb{Z}[\Omega]_{+}}} A_{\boldsymbol{t}}^{y} h(\boldsymbol{n}, \boldsymbol{t}, y)\right\rangle \\
& =\sum_{x, y \in X} \sum_{\substack{\boldsymbol{m}, \boldsymbol{n} \in \mathbb{Z}[\Omega]_{+} \\
\boldsymbol{s}, \boldsymbol{t} \in \mathbb{Z}[\Omega]_{+}}}\left\langle\boldsymbol{T}^{(\boldsymbol{m}-\boldsymbol{n})^{+}} A_{\boldsymbol{s}}^{x} h(\boldsymbol{m}, \boldsymbol{s}, x), \boldsymbol{T}^{(\boldsymbol{m}-\boldsymbol{n})^{-}} A_{\boldsymbol{t}}^{y} h(\boldsymbol{n}, \boldsymbol{t}, y)\right\rangle \\
& =\sum_{x, y \in X} \sum_{\substack{\boldsymbol{m}, \boldsymbol{n} \in \mathbb{Z}[\Omega]_{+} \\
\boldsymbol{s}, \boldsymbol{t} \in \mathbb{Z}[\Omega]_{+}}}\left\langle A_{(\boldsymbol{m}-\boldsymbol{n})^{+}+\boldsymbol{s}}^{x} h(\boldsymbol{m}, \boldsymbol{s}, x), A_{(\boldsymbol{m}-\boldsymbol{n})^{-}+\boldsymbol{t}}^{y} h(\boldsymbol{n}, \boldsymbol{t}, y)\right\rangle .
\end{aligned}
$$

(ii) $\Rightarrow$ (i). Take $\alpha \in \Omega$ and $h_{1}, h_{2} \in \mathcal{D}\left[\mathbb{Z}[\Omega]_{+} \times X\right]$. Define $f: \mathbb{Z}[\Omega]_{+} \times \mathbb{Z}[\Omega]_{+} \times X \rightarrow$ $\mathcal{D}$ by

$$
f(\boldsymbol{m}, \boldsymbol{s}, x)=\left\{\begin{array}{cl}
h_{1}(\boldsymbol{s}, x) & \boldsymbol{m}=\boldsymbol{e}_{\alpha} \\
h_{2}(\boldsymbol{s}, x) & \boldsymbol{m}=2 \boldsymbol{e}_{\alpha} \\
0 & \text { otherwise }
\end{array}\right.
$$

Hence, by (ii), we get

$$
\begin{aligned}
0 & \leqslant \sum_{x, y \in X} \sum_{\substack{\boldsymbol{m}, \boldsymbol{n} \in \mathbb{Z}[\Omega]_{+} \\
\boldsymbol{s}, \boldsymbol{t} \in \mathbb{Z}[\Omega]_{+}}}\left\langle A_{(\boldsymbol{m}-\boldsymbol{n})^{+}+\boldsymbol{s}}^{x} f(\boldsymbol{m}, \boldsymbol{s}, x), A_{(\boldsymbol{m}-\boldsymbol{n})^{-+}+\boldsymbol{t}}^{y} f(\boldsymbol{n}, \boldsymbol{t}, y)\right\rangle \\
& =\sum_{k, l=1}^{2} \sum_{\substack{x, y \in X \\
\boldsymbol{s}, \boldsymbol{t} \in \mathbb{Z}[\Omega]_{+}}}\left\langle A_{(k-l)^{+} \boldsymbol{e}_{\alpha}+\boldsymbol{s}}^{x} h_{k}(\boldsymbol{s}, x), A_{(k-l)^{-} \boldsymbol{e}_{\alpha}+\boldsymbol{t}}^{y} h_{l}(\boldsymbol{t}, y)\right\rangle .
\end{aligned}
$$

According to Lemma 2 there exists a family $\widetilde{\boldsymbol{T}}=\left\{\widetilde{T}_{\alpha}\right\}_{\alpha \in \Omega} \subset \boldsymbol{B}\left(\mathcal{H}_{\mathrm{A}}\right)$ of commuting contractions such that $\widetilde{T}_{\alpha} A_{\boldsymbol{m}}^{x}=A_{\boldsymbol{e}_{\alpha}+\boldsymbol{m}}^{x}$ for all $\alpha \in \Omega, x \in X$ and $\boldsymbol{m} \in \mathbb{Z}[\Omega]_{+}$. Arguing as in the proof of the implication (i) $\Rightarrow$ (ii) we show that for any map $f \in \mathcal{R}_{\mathrm{A}}\left[\mathbb{Z}[\Omega]_{+}\right]$the following inequality holds:

$$
\sum_{\boldsymbol{m}, \boldsymbol{n} \in \mathbb{Z}[\Omega]_{+}}\left\langle\boldsymbol{T}^{(\boldsymbol{m}-\boldsymbol{n})^{+}} f(\boldsymbol{m}), \boldsymbol{T}^{(\boldsymbol{m}-\boldsymbol{n})^{-}} f(\boldsymbol{n})\right\rangle \geqslant 0 .
$$

Exploiting the continuity of $\widetilde{T}_{\alpha}$ and applying the implication (b) $\Rightarrow($ a) of Lemma 1 we deduce that the family $\widetilde{\boldsymbol{T}}$ has a regular unitary dilation. As a consequence, the family $\boldsymbol{T}=\left\{T_{\alpha}\right\}_{\alpha \in \Omega} \subset \boldsymbol{B}(\mathcal{H})$ defined by $T_{\alpha}=\widetilde{T}_{\alpha} \oplus I_{\mathcal{H} \ominus \mathcal{H}_{A}}, \alpha \in \Omega$, has all the required properties listed in (i). 
(i) $\Rightarrow\left(\right.$ iii). Take $h \in \mathcal{D}\left[\mathbb{Z}[\Omega]_{+} \times X\right]$ and a finite subset $u$ of $\Omega$. Define $f \in \mathcal{H}$ by

$$
f=\sum_{\substack{x \in X \\ \boldsymbol{m} \in \mathbb{Z}[\Omega]_{+}}} A_{\boldsymbol{m}}^{x} h(\boldsymbol{m}, x)
$$

Applying the implication (a) $\Rightarrow$ (c) of Lemma 1 and using (1) we get

$$
\begin{aligned}
0 & \leqslant \sum_{v \subseteq u}(-1)^{|v|}\left\|\boldsymbol{T}^{e_{v}} f\right\|^{2}=\sum_{v \subseteq u}(-1)^{|v|}\left\|\sum_{\substack{x \in X \\
\boldsymbol{m} \in \mathbb{Z}[\Omega]_{+}}} \boldsymbol{T}^{\boldsymbol{e}_{v}} A_{\boldsymbol{m}}^{x} h(\boldsymbol{m}, x)\right\|^{2} \\
& =\sum_{v \subseteq u}(-1)^{|v|}\left\|\sum_{\substack{x \in X \\
\boldsymbol{m} \in \mathbb{Z}[\Omega]_{+}}} A_{\boldsymbol{e}_{v}+\boldsymbol{m}}^{x} h(\boldsymbol{m}, x)\right\|^{2} .
\end{aligned}
$$

(iii) $\Rightarrow$ (i). Take $\alpha \in \Omega$. It follows from (iii) with $u \stackrel{\text { df }}{=}\{\alpha\}$ that the condition (iii) of Lemma 2 holds. Hence, by Lemma 2 there exists a unique family $\widetilde{\boldsymbol{T}}=$ $\left\{\widetilde{T}_{\alpha}\right\}_{\alpha \in \Omega} \subset \boldsymbol{B}\left(\mathcal{H}_{\mathrm{A}}\right)$ of commuting contractions such that $\widetilde{T}_{\alpha} A_{\boldsymbol{m}}^{x}=A_{\boldsymbol{e}_{\alpha}+\boldsymbol{m}}^{x}$ for all $\alpha \in \Omega, \boldsymbol{m} \in \mathbb{Z}[\Omega]_{+}$and $x \in X$. Arguing as in the proof of the implication (i) $\Rightarrow$ (iii) we show that for any finite subset $u$ of $\Omega$ and for any vector $f \in \mathcal{R}_{\mathrm{A}}$ the following inequality holds:

$$
\sum_{v \subseteq u}(-1)^{|v|}\left\|\boldsymbol{T}^{e_{v}} f\right\|^{2} \geqslant 0
$$

Exploiting the continuity of $\widetilde{T}_{\alpha}$ and applying the implication (c) $\Rightarrow$ (a) of Lemma 1 we deduce that the family $\widetilde{\boldsymbol{T}}$ has a regular unitary dilation. As a consequence, the family $\boldsymbol{T}=\left\{T_{\alpha}\right\}_{\alpha \in \Omega} \subset \boldsymbol{B}(\mathcal{H})$ defined by $T_{\alpha}=\widetilde{T}_{\alpha} \oplus I_{\mathcal{H} \ominus \mathcal{H}_{\mathrm{A}}}, \alpha \in \Omega$, has all the required properties listed in (i).

Our Theorem 4 includes the solution of the vector moment problem as a particular case (by identifying vectors $h_{\boldsymbol{x}}$ with operators $A_{\boldsymbol{x}}: \mathbb{C} \rightarrow \mathcal{H}$ via $A_{\boldsymbol{x}} \lambda=\lambda h_{\boldsymbol{x}}$, $\lambda \in \mathbb{C}$ ), which generalizes [11, Theorem 3.1] and [13, Theorem C]. More precisely, we get the following.

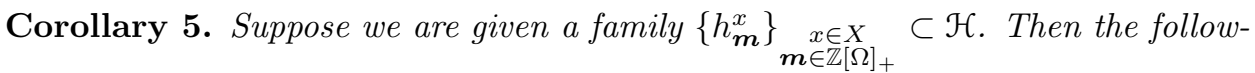
ing conditions are equivalent:

(i) there exists a family $\boldsymbol{T}=\left\{T_{\alpha}\right\}_{\alpha \in \Omega} \subset \boldsymbol{B}(\mathcal{H})$ of commuting contractions having a regular unitary dilation and such that

$$
h_{\boldsymbol{m}}^{x}=\boldsymbol{T}^{\boldsymbol{m}} h_{\mathbf{0}}^{x}, \quad \boldsymbol{m} \in \mathbb{Z}[\Omega]_{+}, x \in X
$$

(ii) for any map $c \in \mathbb{C}\left[\mathbb{Z}[\Omega]_{+} \times \mathbb{Z}[\Omega]_{+} \times X\right]$ the following inequality holds:

$$
\sum_{x, y \in X} \sum_{\substack{\boldsymbol{m}, \boldsymbol{n} \in \mathbb{Z}[\Omega]_{+} \\ \boldsymbol{s}, \boldsymbol{t} \in \mathbb{Z}[\Omega]_{+}}} c(\boldsymbol{m}, \boldsymbol{s}, x) \overline{c(\boldsymbol{n}, \boldsymbol{t}, y)}\left\langle h_{(\boldsymbol{m}-\boldsymbol{n})^{++}}^{x}, h_{(\boldsymbol{m}-\boldsymbol{n})^{-+}+\boldsymbol{t}}^{y}\right\rangle \geqslant 0
$$

(iii) for any map $c \in \mathbb{C}\left[\mathbb{Z}[\Omega]_{+} \times X\right]$ and for any finite subset $u$ of $\Omega$ the following inequality holds:

$$
\sum_{v \subseteq u}(-1)^{|v|}\left\|\sum_{\substack{x \in X \\ m \in \mathbb{Z}[\Omega]_{+}}} c(\boldsymbol{m}, x) h_{\boldsymbol{e}_{v}+\boldsymbol{m}}^{x}\right\|^{2} \geqslant 0 .
$$

It turns out that Corollaries 9 and 10 of 8 , with the appropriate changes, are also valid in the case of the regular dilation. 
Corollary 6. Suppose that $\left\{A_{\sigma, \boldsymbol{m}}^{x}\right\} \underset{\boldsymbol{m} \in \mathbb{Z} \in[\Omega]_{+}}{ } \subset \boldsymbol{L}(\mathcal{D}, \mathcal{H}), \sigma \in \Sigma$, is a net of families of operators and $\left\{A_{\boldsymbol{m}}^{x}\right\}_{\boldsymbol{m} \in \mathbb{Z}[\Omega]_{+}} \subset \boldsymbol{L}(\mathcal{D}, \mathcal{H})$ is a family such that

$$
\lim _{\sigma \in \Sigma} A_{\sigma, \boldsymbol{m}}^{x} h=A_{\boldsymbol{m}}^{x} h, \quad h \in \mathcal{D}, \boldsymbol{m} \in \mathbb{Z}[\Omega]_{+}, x \in X .
$$

If for every $\sigma \in \Sigma$, there exists a family $\boldsymbol{T}_{\sigma}=\left\{T_{\sigma, \alpha}\right\}_{\alpha \in \Omega} \subset \boldsymbol{B}(\mathcal{H})$ of commuting contractions having a regular unitary dilation and such that $A_{\sigma, \boldsymbol{m}}^{x}=\boldsymbol{T}_{\sigma}^{\boldsymbol{m}} A_{\sigma, \mathbf{0}}^{x}$ for all $\boldsymbol{m} \in \mathbb{Z}[\Omega]_{+}$and $x \in X$, then there exists a family $\boldsymbol{T}=\left\{T_{\alpha}\right\}_{\alpha \in \Omega} \subset \boldsymbol{B}(\mathcal{H})$ of commuting contractions having a regular unitary dilation and such that

$$
A_{\boldsymbol{m}}^{x}=\boldsymbol{T}^{\boldsymbol{m}} A_{\mathbf{0}}^{x}, \quad \boldsymbol{m} \in \mathbb{Z}[\Omega]_{+}, x \in X .
$$

Proof. By Theorem 4 , the family $\left\{A_{\sigma, \boldsymbol{m}}^{x}\right\} \underset{\boldsymbol{m} \in \mathbb{Z}[\Omega]_{+}}{x \in X}$ satisfies condition (ii) of Theorem 4. After passage to the limit with $\sigma$, we see that the limit family $\left\{A_{\boldsymbol{m}}^{x}\right\} \underset{\substack{x \in X \\ \boldsymbol{m} \in \mathbb{Z}[\Omega]_{+}}}{\substack{+ \\ \text { (n) }}}$ satisfies the same condition, which by Theorem 4 completes the proof.

Corollary 7. If $\left\{A_{\boldsymbol{m}}^{x}\right\} \underset{\substack{x \in X \\ \boldsymbol{m} \in \mathbb{Z}[\Omega]_{+}}}{ } \subset \boldsymbol{L}(\mathcal{D}, \mathcal{H})$, then the following are equivalent:

(i) there exists a family $\boldsymbol{T}=\left\{T_{\alpha}\right\}_{\alpha \in \Omega} \subset \boldsymbol{B}(\mathcal{H})$ of commuting contractions having a regular unitary dilation and such that (10) holds;

(ii) for every finite subset $\Xi$ of $\Omega$, there exists a family $\boldsymbol{T}_{\Xi}=\left\{T_{\Xi, \alpha}\right\}_{\alpha \in \Xi} \subset$ $\boldsymbol{B}(\mathcal{H})$ of commuting contractions having a regular unitary dilation and such that $A_{\boldsymbol{m}}^{x}=\boldsymbol{T}_{\Xi}^{\boldsymbol{m}} A_{\mathbf{0}}^{x}$ for all $\boldsymbol{m} \in \mathbb{Z}[\Xi]_{+}$and $x \in X$;

(iii) for every finite-dimensional linear subspace $\mathcal{C}$ of $\mathcal{D}$, there exists a family $\boldsymbol{T}_{\mathcal{C}}=\left\{T_{\mathfrak{e}, \alpha}\right\}_{\alpha \in \Omega} \subset \boldsymbol{B}(\mathcal{H})$ of commuting contractions having a regular unitary dilation and such that $\left.A_{\boldsymbol{m}}^{x}\right|_{\mathcal{e}}=\left.\boldsymbol{T}_{\mathfrak{e}}^{\boldsymbol{m}} A_{\mathbf{0}}^{x}\right|_{\mathrm{e}}$ for all $\boldsymbol{m} \in \mathbb{Z}[\Omega]_{+}$and $x \in X$

(iv) for every finite subset $\Xi$ of $\Omega$ and for every finite-dimensional linear subspace $\mathcal{C}$ of $\mathcal{D}$, there exists a family $\boldsymbol{T}_{\Xi, \mathcal{C}}=\left\{T_{\Xi, \mathcal{C}, \alpha}\right\}_{\alpha \in \Xi} \subset \boldsymbol{B}(\mathcal{H})$ of commuting contractions having a regular unitary dilation and such that $\left.A_{m}^{x}\right|_{e}=$ $\left.\boldsymbol{T}_{\Xi, \mathrm{e}}^{\boldsymbol{m}} A_{\mathbf{0}}^{x}\right|_{\mathrm{e}}$ for all $\boldsymbol{m} \in \mathbb{Z}[\Xi]_{+}$and $x \in X$.

Proof. Since $\mathbb{Z}[\Omega]=\bigcup\{\mathbb{Z}[\Xi]: \Xi$ a finite subset of $\Omega\}$ we may apply any of the conditions (ii) or (iii) of Theorem 4 to get the equivalences (i) $\Leftrightarrow($ ii) and (i) $\Leftrightarrow$ (iii). The equivalence (i) $\Leftrightarrow$ (iv) follows from the equivalences (i) $\Leftrightarrow($ ii $) \Leftrightarrow($ iii).

4. Let us recall (cf. [8, Theorem 7]) that a family $\left\{A_{\boldsymbol{m}}\right\}_{\boldsymbol{m} \in \mathbb{Z}[\Omega]_{+}} \subset \boldsymbol{L}(\mathcal{D}, \mathcal{H})$ of operators is of the form

$$
A_{\boldsymbol{m}}=\boldsymbol{T}^{\boldsymbol{m}} A_{\mathbf{0}}, \quad \boldsymbol{m} \in \mathbb{Z}[\Omega]_{+},
$$

where $\boldsymbol{T}=\left\{T_{\alpha}\right\}_{\alpha \in \Omega} \subset \boldsymbol{B}(\mathcal{H})$ is a family of commuting contractions having a unitary dilation (not necessarily regular ones), if and only if the following condition holds:

(ii $\left.{ }^{\star}\right)$ for any integer $c \geqslant 1$ and for all maps $h_{1}, \ldots, h_{c} \in \mathcal{D}\left[\mathbb{Z}[\Omega]_{+}^{2}\right]$ such that

$$
\begin{aligned}
\sum_{j=1}^{c} \sum_{\substack{\boldsymbol{m}, \boldsymbol{n} \in \mathbb{Z}[\Omega]_{+} \\
\boldsymbol{m}-\boldsymbol{n}=\boldsymbol{u}}} \sum_{\boldsymbol{s}, \boldsymbol{t} \in \mathbb{Z}[\Omega]_{+}}\left(A_{\boldsymbol{s}} h_{j}(\boldsymbol{m}, \boldsymbol{s})\right) \otimes\left(A_{\boldsymbol{t}} h_{j}(\boldsymbol{n}, \boldsymbol{t})\right)=0, & \\
\boldsymbol{u} & \in\left(\mathbb{Z}[\Omega] \backslash\left(\mathbb{Z}[\Omega]_{+} \cup\left(-\mathbb{Z}[\Omega]_{+}\right)\right)\right),
\end{aligned}
$$


the following inequality holds:

$$
\sum_{j=1}^{c} \sum_{\substack{\boldsymbol{m}, \boldsymbol{n} \in \mathbb{Z}[\Omega]_{+} \\ \boldsymbol{s}, \boldsymbol{t} \in \mathbb{Z}[\Omega]_{+}}}\left\langle A_{(\boldsymbol{m}-\boldsymbol{n})^{+}+\boldsymbol{s}} h_{j}(\boldsymbol{m}, \boldsymbol{s}), A_{(\boldsymbol{m}-\boldsymbol{n})^{-}+\boldsymbol{t}} h_{j}(\boldsymbol{n}, \boldsymbol{t})\right\rangle \geqslant 0 .
$$

Comparing it to our condition (ii) of Theorem 4 (with $\operatorname{card}(X)=1)$, it is evident that the condition (ii) of Theorem 4 implies $\left(\right.$ ii $\left.^{\star}\right)$.

\section{ACKNOWLEDGMENTS}

The author would like to thank Professor Jan Stochel for helpful discussions concerning the subject of the paper. I would like to also thank the referee for helpful comments.

\section{REFERENCES}

[1] S. Brehmer, Über vertauschbare Kontraktionen des Hilbertschen Raumes, Acta Sci. Math. (Szeged) 22 (1961), 106-111. MR0131169 (24:A1023a)

[2] R. E. Curto, F. H. Vasilescu, Standard operator models in the polydisc, Indiana Univ. Math. J. 42 (1993), 791-810. MR.1254118 (94k:47008)

[3] R. E. Curto, F. H. Vasilescu, Standard operator models in the polydisc II, Indiana Univ. Math. J. 44 (1995), 727-746. MR.1375346 (96k:47009)

[4] P. Gãvruţã, D. Pãunescu, Sebestyén's moment problem and regular dilations, Acta Math. Hungar. 94 (2002), 223-232. MR.1905727 (2003g:47014)

[5] D. Gaspar, N. Suciu, On the intertwinings of regular dilations, Ann. Pol. Math. 66 (1997), 105-121. MR.1438333 (98c:47008)

[6] D. Gaspar, N. Suciu, On the geometric structure of regular dilations, Oper. Theory, Adv. Appl. 103 (1998), 105-120. MR1635001 (99h:47012)

[7] I. Halperin, Sz.-Nagy-Brehmer dilations, Acta Sci. Math. (Szeged) 23 (1962), 279-289. MR.0156196 (26:6127a)

[8] Z. J. Jabłoński, J. Stochel, F. H. Szafraniec, Unitary propagation of operator data, to appear in Proc. Edinburgh Math. Soc.

[9] Y. Kakihara, Multidimensional Second Order Stochastic Processes, Series on Multivariate Analysis 2, World Scientific Publ. Co., Inc., River Edge, NJ, 1997. MR.1625379 (2000g:60061)

[10] A. Olofsson, Operator-valued $n$-harmonic measure in the polydisc, Studia Math. 163 (2004), 203-216. MR2078443 (2005f:47011)

[11] D. Popovici, Bi-dimensional moment problems and regular dilations, Operator Theory and Indefinite Inner Product Spaces, 257-274, Oper. Theory Adv. Appl., 163, Birkhäuser, Basel, 2006.

[12] D. Popovici, Dilatable solutions for some operator moment problems, preprint 2005.

[13] D. Popovici, Z. Sebestyén, Sebestyén moment problem: the multi-dimensional case, Proc. Amer. Math. Soc. 132 (2004), 1029-1035. MR2045418 (2004m:47030)

[14] Yu. A. Rozanov, Spectral analysis of abstract functions, Theory Probab. Appl. 4 (1959), 271-287. MR0123357 (23:A685)

[15] Z. Sebestyén, Moment theorems for operators of Hilbert space, Acta Sci. Math. (Szeged) 44 (1982), 165-171. MR0660523 (84f:47004)

[16] Z. Sebestyén, Moment theorems for operators on Hilbert space. II, Acta Sci. Math. (Szeged) 47 (1984), 101-106. MR0755567 (86b:47065)

[17] Z. Sebestyén, D. Popovici, Positive definite functions and Sebestyén's operator moment problem, Glasgow Math. J. 47 (2005), 471-488. MR2202060(2006i:47031)

[18] B. Sz.-Nagy, Sur les contractions de l'espace de Hilbert, Acta Sci. Math. (Szeged) 15 (1953), 87-92. MR0058128 (15:326d)

[19] B. Sz.-Nagy, Bemerkungen zur vorstehenden Arbeit des Herrn S. Brehmer, Acta Sci. Math. (Szeged) 22 (1961), 112-114. MR0131170(24:A1023b)

[20] B. Sz.-Nagy, C. Foiaş, Harmonic analysis of operators on Hilbert space, Akadémiai Kiadó, Budapest 1970. 
[21] D. Timotin, Regular dilations and models for multicontractions, Indiana Univ. Math. J. 47 (1998), 671-684. MR1647873 (2000c:47019)

Instytut Matematyki, Uniwersytet Jagielloński, Ul. Reymonta 4, PL-30059 Kraków, POLAND

E-mail address: jablonsk@im.uj.edu.pl 